Acta Crystallographica Section E

Structure Reports

Online

ISSN 1600-5368

\section{4-Chlorobenzoyl-meso-octamethyl- calix[2]pyrrolidino[2]pyrrole: an acyl chloride derivative of a partially reduced calix[4] pyrrole}

\author{
Guillaume Journot, ${ }^{\mathrm{a}}$ Reinhard Neier ${ }^{\mathrm{a} *}$ and Helen Stoeckli- \\ Evans $^{\text {b* }}$
}

anstitute of Chemistry, University of Neuchâtel, Avenue de Bellevaux 51, CH-2000 Neuchâtel, Switzerland, and ${ }^{\mathbf{b}}$ Institute of Physics, University of Neuchâtel, Rue Emile-Argand 11, CH-2000 Neuchâtel, Switzerland

Correspondence e-mail: reinhard.neier@unine.ch, helen.stoeckli-evans@unine.ch

Received 15 February 2012; accepted 16 February 2012

Key indicators: single-crystal X-ray study; $T=173 \mathrm{~K}$; mean $\sigma(\mathrm{C}-\mathrm{C})=0.003 \AA$; $R$ factor $=0.053 ; w R$ factor $=0.102 ;$ data-to-parameter ratio $=15.5$.

In the title compound, $\mathrm{C}_{35} \mathrm{H}_{47} \mathrm{ClN}_{4} \mathrm{O}$, the two pyrrolidine rings have envelope conformations. The conformation of the macrocycle is stabilized by $\mathrm{N}-\mathrm{H} \cdots \mathrm{N}$ hydrogen bonds and a $\mathrm{C}-\mathrm{H} \cdots \mathrm{N}$ interaction. The benzoyl ring is inclined to the adjacent pyrrole ring by $11.66(11)^{\circ}$, with a centroid-centroid distance of 3.7488 (13) A. In the crystal, molecules are linked by $\mathrm{N}-\mathrm{H} \cdots \mathrm{O}$ hydrogen bonds into helical chains propagating in $[010]$ and $\mathrm{C}-\mathrm{H} \cdots \mathrm{O}$ and $\mathrm{C}-\mathrm{H} \cdots \pi$ interactions are also observed.

\section{Related literature}

For the heterogeneous catalytic hydrogenation of meso-octamethylcalix[4]pyrrole, which gave meso-octamethylcalix[2]pyrrole[2]pyrrolidine, see: Blangy et al. (2009). For $N$-acylation of pyrrolidines, using substituted benzoyl chlorides, see: Journot et al. (2012a); Zhang et al. (2009). For the synthesis and reactivity of the title compound, see: Journot \& Neier (2012). For the crystal structures of similar compounds, see: Journot et al. $(2012 b, c, d, e)$.

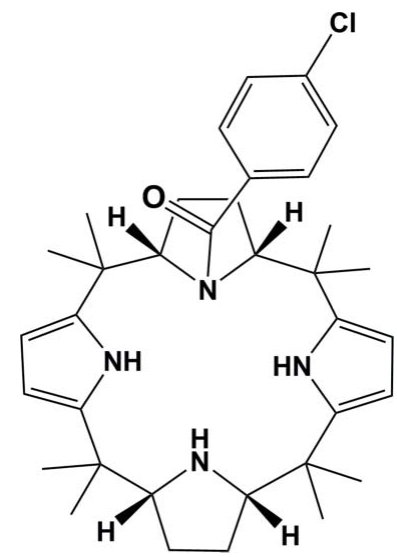

\section{Experimental}

Crystal data

$\mathrm{C}_{35} \mathrm{H}_{47} \mathrm{ClN}_{4} \mathrm{O}$

$M_{r}=575.22$

Monoclinic, $P 2_{1} / n$

$a=10.3224$ (6) $\AA$

$b=12.0389$ (4) $\AA$

$c=25.3311$ (13)

$\beta=96.798$ (4)

$V=3125.8(3) \AA^{3}$

$Z=4$

Mo $K \alpha$ radiation

$\mu=0.16 \mathrm{~mm}^{-1}$

$T=173 \mathrm{~K}$

$0.45 \times 0.42 \times 0.40 \mathrm{~mm}$

\section{Data collection}

Stoe IPDS 2 diffractometer

Absorption correction: multi-scan

(MULscanABS in PLATON;

Spek, 2009)

$T_{\min }=0.973, T_{\max }=1.000$

Refinement

$R\left[F^{2}>2 \sigma\left(F^{2}\right)\right]=0.053$

$w R\left(F^{2}\right)=0.102$

$S=1.03$

5906 reflections

381 parameters

1 restraint

32665 measured reflections 5906 independent reflections 4215 reflections with $I>2 \sigma(I)$ $R_{\text {int }}=0.076$

$\mathrm{H}$ atoms treated by a mixture of independent and constrained refinement

$\Delta \rho_{\max }=0.24{\mathrm{e} \AA^{-3}}^{-3}$

$\Delta \rho_{\min }=-0.34{\mathrm{e} \AA^{-3}}^{-3}$

Table 1

Hydrogen-bond geometry $\left(\AA{ }^{\circ}\right)$.

$C g 1$ is the centroid of pyrrole ring N2/C3/C4/C25/C26; $C g 2$ is the centroid of the benzene ring $\mathrm{C} 30-\mathrm{C} 35$.

\begin{tabular}{lllll}
\hline$D-\mathrm{H} \cdots A$ & $D-\mathrm{H}$ & $\mathrm{H} \cdots A$ & $D \cdots A$ & $D-\mathrm{H} \cdots A$ \\
\hline $\mathrm{N} 2-\mathrm{H} 2 \cdots \mathrm{N} 3$ & 0.88 & 2.31 & $2.865(2)$ & 121 \\
$\mathrm{~N} 4-\mathrm{H} 4 \cdots \mathrm{N} 3$ & 0.88 & 2.55 & $3.051(2)$ & 117 \\
$\mathrm{C} 15-\mathrm{H} 15 A \cdots \mathrm{N} 2$ & 0.98 & 2.52 & $3.488(3)$ & 171 \\
$\mathrm{C} 15-\mathrm{H} 15 A \cdots C g 1$ & 0.98 & 2.40 & $3.301(2)$ & 152 \\
$\mathrm{~N} 3-\mathrm{H} 3 N \cdots \mathrm{O} 1^{\mathrm{i}}$ & $0.882(18)$ & $2.257(18)$ & $3.105(2)$ & $161.1(18)$ \\
$\mathrm{C} 23-\mathrm{H} 23 B \cdots \mathrm{O} 1^{\mathrm{i}}$ & 0.98 & 2.53 & $3.495(3)$ & 168 \\
$\mathrm{C} 27-\mathrm{H} 27 C \cdots C g 2^{\mathrm{i}}$ & 0.98 & 2.82 & $3.702(2)$ & 150 \\
\hline
\end{tabular}

Symmetry code: (i) $-x+\frac{3}{2}, y-\frac{1}{2},-z+\frac{1}{2}$.

Data collection: $X$-AREA (Stoe \& Cie, 2009); cell refinement: $X$-AREA; data reduction: $X$-RED32 (Stoe \& Cie, 2009); program(s) used to solve structure: SHELXS97 (Sheldrick, 2008); program(s) used to refine structure: SHELXL97 (Sheldrick, 2008); molecular graphics: PLATON (Spek, 2009); software used to prepare material for publication: SHELXL97, PLATON and publCIF (Westrip, 2010).

HSE thanks the staff of the XRD Application Laboratory, CSEM, Neuchâtel for access to the X-ray diffraction equipment.

Supplementary data and figures for this paper are available from the IUCr electronic archives (Reference: ZQ2154).

\title{
References
}

Blangy, V., Heiss, C., Khlebnikov, V., Letondor, C., Stoeckli-Evans, H. \& Neier, R. (2009). Angew. Chem. Int. Ed. 48, 1688-1691.

Journot, G. \& Neier, R. (2012). In preparation.

Journot, G., Neier, R. \& Stoeckli-Evans, H. (2012a). Acta Cryst. C68, o119o122.

Journot, G., Neier, R. \& Stoeckli-Evans, H. (2012b). Acta Cryst. E68, o929o930.

Journot, G., Neier, R. \& Stoeckli-Evans, H. (2012c). Private communication (deposition number CCDC-866917). CCDC, Cambridge, England. 


\section{organic compounds}

Journot, G., Neier, R. \& Stoeckli-Evans, H. (2012d). Private communication (deposition number CCDC-866918). CCDC, Cambridge, England.

Journot, G., Neier, R. \& Stoeckli-Evans, H. (2012e). Private communication (deposition number CCDC-866919). CCDC, Cambridge, England.

Sheldrick, G. M. (2008). Acta Cryst. A64, 112-122.

Spek, A. L. (2009). Acta Cryst. D65, 148-155.
Stoe \& Cie (2009). $X$-AREA and X-RED32. Stoe \& Cie GmbH, Darmstadt, Germany.

Westrip, S. P. (2010). J. Appl. Cryst. 43, 920-925.

Zhang, L., Wang, X.-J., Wang, J., Grinberg, N., Krishnamurthy, D. K. \& Senanayake, C. H. (2009). Tetrahedron Lett. 50, 2964-2966. 


\section{supporting information}

Acta Cryst. (2012). E68, o976-o977 [https://doi.org/10.1107/S1600536812007003]

\section{4-Chlorobenzoyl-meso-octamethylcalix[2]pyrrolidino[2] pyrrole: an acyl chloride derivative of a partially reduced calix[4] pyrrole}

\section{Guillaume Journot, Reinhard Neier and Helen Stoeckli-Evans}

\section{S1. Comment}

We have recently reported the access to new macrocycles by heterogeneous catalytic hydrogenation of meso-octamethylcalix[4]pyrrole, which gave meso-octamethylcalix[2]pyrrole[2]pyrrolidine (1 in Fig. 3) [Blangy et al., 2009]. It was decided to investigate the nucleophilicity of this new macrocycle, which showed interesting reactivity (Journot \& Neier, 2012), by reacting different substituted benzoyl chlorides with the macrocycle under smooth conditions [Journot et al., 2012a; Zhang et al., 2009]. Herein, we report on the synthesis and crystal structure of the title 4-chlorobenzoyl derivative, one of five compounds that have been studied by X-ray diffraction analysis (Journot et al., 2012b,c,d,e). The molecular structure of the title compound is given in Fig. 1. The two pyrrolidine rings $(\mathrm{N} 1, \mathrm{C} 1, \mathrm{C} 12-\mathrm{C} 14)$ and $(\mathrm{N} 3, \mathrm{C} 6, \mathrm{C} 7, \mathrm{C} 21, \mathrm{C} 22)$ have envelope conformations with, respectively, atoms $\mathrm{C} 13$ and $\mathrm{C} 6$ as the flaps. The conformation of the macrocycle is stabilized by intramolecular $\mathrm{N}-\mathrm{H} \cdots \mathrm{N}$ hydrogen bonds involving atom $\mathrm{N} 3$ and the two pyrrole $\mathrm{H}$ atoms, $\mathrm{H} 2$ and $\mathrm{H} 4$ (Fig. 1 and Table 1). The benzoyl ring $(\mathrm{C} 30-\mathrm{C} 35)$ is inclined to the pyrrole ring (N4,C9,C10,C17,C18) by $11.66(11)^{\circ}$, with a centroid-to-centroid distance of 3.7488 (13) $\AA$. The methyl group C15 is also in close contact with the pyrrole ring $(\mathrm{N} 2, \mathrm{C} 3, \mathrm{C} 4, \mathrm{C} 25, \mathrm{C} 26)$, with a short $\mathrm{C} 15-\mathrm{H} 15 \mathrm{~A} \cdots \mathrm{N} 2$ interaction and a $\mathrm{C} 15-$ H15A $\cdots$ centroid distance of 3.301 (2) [see Table 1].

In the crystal, molecules are linked via an $\mathrm{N}-\mathrm{H} \cdots \mathrm{O}$ hydrogen bond, involving the $\mathrm{N} 3$ pyrrolidine $\mathrm{H}$-atom $(\mathrm{H} 3 \mathrm{~N})$ and the benzoyl $\mathrm{O}$ atom (O1), leading to the formation of helical chains propagating along [010] - see Fig. 2 and Table 1. The same $\mathrm{O}$ atom is involved in a $\mathrm{C}-\mathrm{H} \cdots \mathrm{O}$ contact with methyl group $\mathrm{C} 23$. A $\mathrm{C}-\mathrm{H} \cdots \pi$ interaction is also observed, involving the methyl group $\mathrm{C} 27$ and the benzoyl ring (C30 - C35) [see Table 1].

The overall geometry and crystal packing is very similar to that reported for the 4-methoxybenzoyl derivative (Journot et al., 2012b), and the 4-nitrobenzoyl (Journot et al., 2012d) and 4-methylbenzoyl (Journot et al., 2012e) derivatives. The benzoyl derivative (Journot et al., 2012c) crystallized, in the trigonal space group $R-3$, as a partial $\left(0.25 \mathrm{H}_{2} \mathrm{O}\right)$ hydrate, and forms hydrogen bonded chains propagating along [001].

Footnote to Table 1: $C g 1$ is the centroid of pyrrole ring (N2,C3,C4,C25,C26); $C g 2$ is the centroid of the benzene ring (C30- 35$)$.

\section{S2. Experimental}

The general procedure for the $N$-acylation of meso-octamethylcalix[2]pyrrolidino[2]pyrrole (1) is illustrated in Fig. 3. A two-necked flask fitted with a gas inlet and containing a stirrer bar was charged with $100 \mathrm{mg}(0.23 \mathrm{mmol})$ of meso-octamethylcalix[2]pyrrolidino[2]pyrrole (1), 4-chlorobenzoyl chloride (2c) (53.61 $\mu \mathrm{L}, 0.48 \mathrm{mmol})$, potassium carbonate (70 $\mathrm{mg}, 0.48 \mathrm{mmol})$ in THF $(5 \mathrm{ml})$ and acetonitrile $(2.5 \mathrm{ml})$. The reaction vessel was flushed with argon and sealed with a septum. After $15 \mathrm{~min}$. a precipitate appeared and the reaction mixture was stirred for $2 \mathrm{~h}$ room temperature. $10 \%$ sodium carbonate was then added and the reaction mixture was extracted with dichloromethane. The organic layer was washed 
successively with two $\times 10 \%$ sodium carbonate and saturated brine. The organic layer was dried with sodium sulfate, and the solvents were removed under vacuum. The residue was purified by column chromatography $\left(\mathrm{SiO}_{2}, \mathrm{CH}_{2} \mathrm{Cl}_{2} / \mathrm{MeOH}\right.$ 97/3) to yield $131.6 \mathrm{mg}(75.6 \%)$ of colourless crystals of the title compound (3c). Melting point: $501 \mathrm{~K}$. HRMS calcd. for $\mathrm{C}_{35} \mathrm{H}_{47} \mathrm{ClN}_{4} \mathrm{O}^{+} \mathrm{H}^{+}$575.3511; found 575.3510. Further synthetic and spectroscopic data have been reported elsewhere (Journot \& Neier, 2012).

\section{S3. Refinement}

The $\mathrm{NH} \mathrm{H}$-atoms were located in a difference electron-density map. H-atom $\mathrm{H} 3 \mathrm{~N}$ was freely refined while the other $\mathrm{NH}$ $\mathrm{H}$-atoms and the $\mathrm{C}$-bound $\mathrm{H}$-atoms were included in calculated positions and treated as riding atoms: $\mathrm{N}-\mathrm{H}=0.88 \AA, \mathrm{C}$ $-\mathrm{H}=0.95 \AA$ for $\mathrm{CH}$-allyl and $\mathrm{CH}$-aromatic $\mathrm{H}$ atoms, and 1.00, 0.99 and $0.98 \AA$, for methine, methylene and methyl $\mathrm{H}$ atoms, respectively, with $U_{\text {iso }}(\mathrm{H})=\mathrm{k} \times U_{\text {eq }}(\mathrm{N}, C)$, where $\mathrm{k}=1.5$ for $\mathrm{CH}_{3} \mathrm{H}$-atoms, and 1.2 for the other $\mathrm{H}$-atoms. 


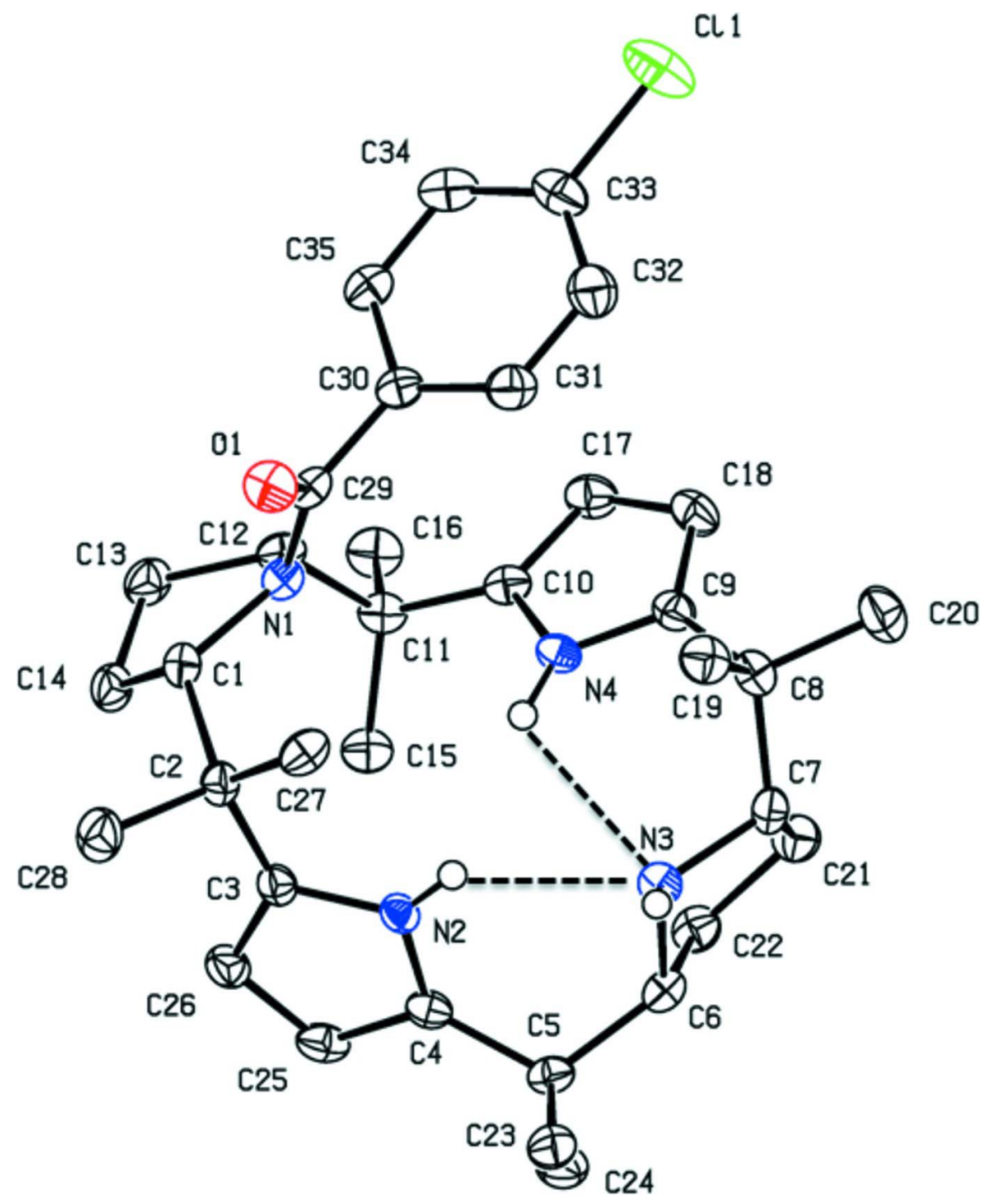

Figure 1

A view of the molecular structure of the title compound, with the numbering scheme and displacement ellipsoids drawn at the $50 \%$ probability level. The $\mathrm{N}-\mathrm{H} \cdots \mathrm{N}$ hydrogen bonds are shown as dashed lines (see Table 1 for details; the $\mathrm{C}$ bound $\mathrm{H}$ atoms have been omitted for clarity). 


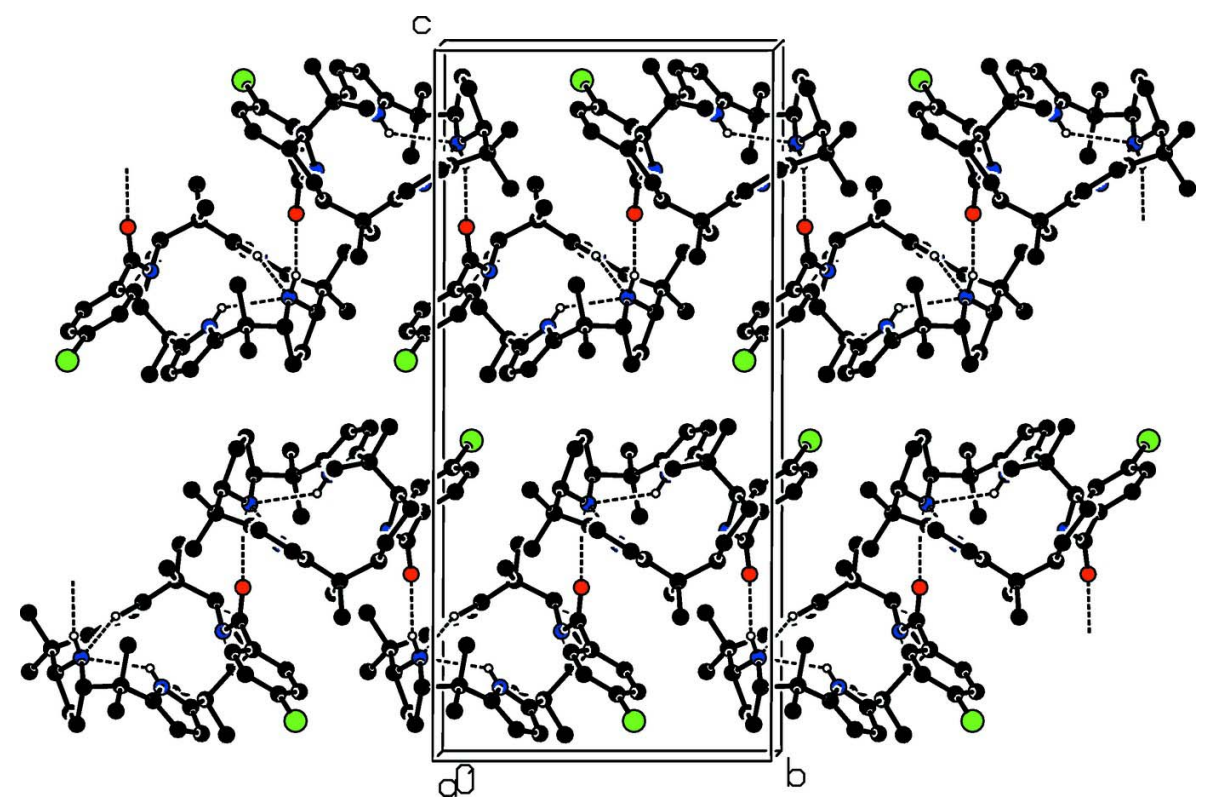

Figure 2

A view along the $a$ axis of the crystal packing of the title compound. The $\mathrm{N}-\mathrm{H} \cdots \mathrm{N}$ and $\mathrm{N}-\mathrm{H} \cdots \mathrm{O}$ hydrogen bonds are shown as dashed lines (see Table 1 for details; the C-bound $\mathrm{H}$ atoms have been omitted for clarity).

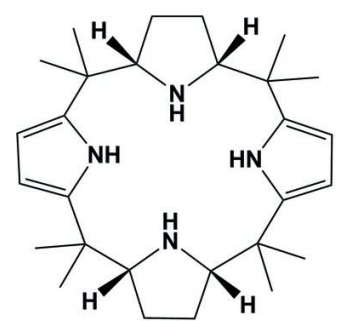

1<smiles>O=C(Cl)c1ccc(Br)cc1</smiles>

2a $\quad R=H$

$2 \mathrm{~b} \quad \mathrm{R}=\mathrm{NO}_{2}$

2c $\mathrm{R}=\mathrm{Cl}$

2d $\mathrm{R}=\mathrm{CH}_{3}$

2e $\mathrm{R}=\mathrm{OCH}_{3}$

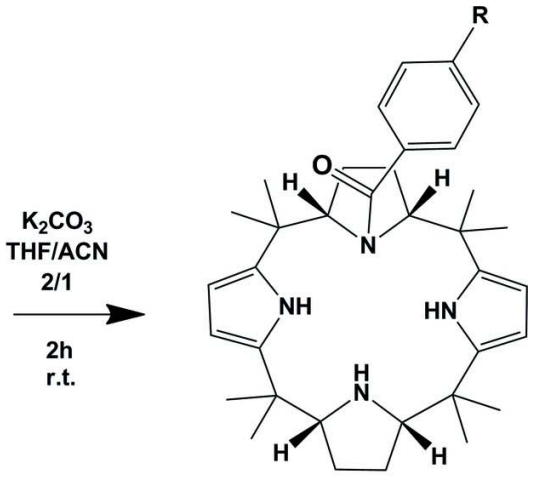

$3 a-3 e$

Figure 3

The general procedure for the $N$-acylation of meso-octamethylcalix[2]pyrrolidino[2]pyrrole (1).

21-[(4-chlorophenyl)carbonyl]-2,2,7,7,12,12,17,17-octamethyl-21,22,23,24-

tetraazapentacyclo[16.2.1.1 $\left.1^{3,6} \cdot 1^{8,11} \cdot 1^{13,16}\right]$ tetracosa-3,5,13,15-tetraene

Crystal data

$\mathrm{C}_{35} \mathrm{H}_{47} \mathrm{ClN}_{4} \mathrm{O}$

$M_{r}=575.22$

Monoclinic, $P 2{ }_{1} / n$

Hall symbol: -P 2 yn

$a=10.3224(6) \AA$

$b=12.0389(4) \AA$

$c=25.3311(13) \AA$ $\beta=96.798(4)^{\circ}$

$V=3125.8(3) \AA^{3}$

$Z=4$

$F(000)=1240$

$D_{\mathrm{x}}=1.222 \mathrm{Mg} \mathrm{m}^{-3}$

Melting point: $501 \mathrm{~K}$

Mo $K \alpha$ radiation, $\lambda=0.71073 \AA$ 
Cell parameters from 16706 reflections

$\theta=1.6-26.1^{\circ}$

$\mu=0.16 \mathrm{~mm}^{-1}$

\section{Data collection}

Stoe IPDS 2 diffractometer

Radiation source: fine-focus sealed tube Graphite monochromator

$\varphi+\omega$ scans

Absorption correction: multi-scan

(MULscanABS in PLATON; Spek, 2009)

$T_{\min }=0.973, T_{\max }=1.000$

\section{Refinement}

Refinement on $F^{2}$

Least-squares matrix: full

$R\left[F^{2}>2 \sigma\left(F^{2}\right)\right]=0.053$

$w R\left(F^{2}\right)=0.102$

$S=1.03$

5906 reflections

381 parameters

1 restraint

Primary atom site location: structure-invariant direct methods
$T=173 \mathrm{~K}$

Block, colourless

$0.45 \times 0.42 \times 0.40 \mathrm{~mm}$

32665 measured reflections

5906 independent reflections

4215 reflections with $I>2 \sigma(I)$

$R_{\text {int }}=0.076$

$\theta_{\max }=25.6^{\circ}, \theta_{\min }=1.6^{\circ}$

$h=-12 \rightarrow 12$

$k=-14 \rightarrow 14$

$l=-30 \rightarrow 30$

Secondary atom site location: difference Fourier map

Hydrogen site location: inferred from neighbouring sites

$\mathrm{H}$ atoms treated by a mixture of independent and constrained refinement

$w=1 /\left[\sigma^{2}\left(F_{\mathrm{o}}^{2}\right)+(0.0461 P)^{2}+0.1416 P\right]$ where $P=\left(F_{\mathrm{o}}{ }^{2}+2 F_{\mathrm{c}}{ }^{2}\right) / 3$

$(\Delta / \sigma)_{\max }<0.001$

$\Delta \rho_{\max }=0.24 \mathrm{e} \AA^{-3}$

$\Delta \rho_{\min }=-0.34$ e $\AA^{-3}$

\section{Special details}

Geometry. Bond distances, angles etc. have been calculated using the rounded fractional coordinates. All su's are estimated from the variances of the (full) variance-covariance matrix. The cell e.s.d.'s are taken into account in the estimation of distances, angles and torsion angles

Refinement. Refinement of $F^{2}$ against ALL reflections. The weighted $R$-factor $w R$ and goodness of fit $S$ are based on $F^{2}$, conventional $R$-factors $R$ are based on $F$, with $F$ set to zero for negative $F^{2}$. The threshold expression of $F^{2}>\sigma\left(F^{2}\right)$ is used only for calculating $R$-factors(gt) $e t c$. and is not relevant to the choice of reflections for refinement. $R$-factors based on $F^{2}$ are statistically about twice as large as those based on $F$, and $R$ - factors based on ALL data will be even larger.

Fractional atomic coordinates and isotropic or equivalent isotropic displacement parameters $\left(\AA^{2}\right)$

\begin{tabular}{lllll}
\hline & $x$ & $y$ & $z$ & $U_{\text {iso }} * / U_{\text {eq }}$ \\
\hline C11 & $1.05031(7)$ & $0.59602(6)$ & $0.05700(3)$ & $0.0518(3)$ \\
O1 & $0.69721(14)$ & $0.42893(12)$ & $0.23989(6)$ & $0.0285(5)$ \\
N1 & $0.54054(15)$ & $0.36573(13)$ & $0.17599(6)$ & $0.0190(5)$ \\
N2 & $0.49236(15)$ & $0.04698(13)$ & $0.19441(6)$ & $0.0190(5)$ \\
N3 & $0.69523(16)$ & $-0.04818(14)$ & $0.14086(7)$ & $0.0205(5)$ \\
N4 & $0.65525(16)$ & $0.18840(13)$ & $0.09895(6)$ & $0.0196(5)$ \\
C1 & $0.45318(19)$ & $0.34058(16)$ & $0.21789(8)$ & $0.0211(6)$ \\
C2 & $0.47134(19)$ & $0.22457(16)$ & $0.24667(8)$ & $0.0211(6)$ \\
C3 & $0.41422(19)$ & $0.12539(16)$ & $0.21499(8)$ & $0.0201(6)$ \\
C4 & $0.41809(19)$ & $-0.03545(16)$ & $0.16818(8)$ & $0.0208(6)$ \\
C5 & $0.4779(2)$ & $-0.14266(16)$ & $0.15089(8)$ & $0.0223(6)$ \\
C6 & $0.59425(19)$ & $-0.12726(16)$ & $0.11840(8)$ & $0.0225(6)$ \\
C7 & $0.7886(2)$ & $-0.03802(17)$ & $0.10096(8)$ & $0.0224(6)$
\end{tabular}




\begin{tabular}{|c|c|c|c|c|}
\hline $\mathrm{C} 8$ & $0.86281(19)$ & $0.07432(17)$ & $0.10394(8)$ & $0.0233(6)$ \\
\hline C9 & $0.77456(19)$ & $0.16747(16)$ & $0.08152(8)$ & $0.0211(6)$ \\
\hline $\mathrm{C} 10$ & $0.59096(19)$ & $0.27254(16)$ & $0.06975(8)$ & $0.0204(6)$ \\
\hline C11 & 0.45514 (19) & $0.31136(16)$ & $0.07862(8)$ & $0.0212(6)$ \\
\hline $\mathrm{C} 12$ & $0.46446(19)$ & $0.39816(16)$ & $0.12436(8)$ & $0.0219(6)$ \\
\hline $\mathrm{C} 13$ & $0.3355(2)$ & $0.43797(17)$ & $0.14288(9)$ & $0.0275(7)$ \\
\hline C14 & $0.3152(2)$ & $0.36450(17)$ & $0.19048(9)$ & $0.0255(7)$ \\
\hline $\mathrm{C} 15$ & $0.3680(2)$ & $0.21172(17)$ & $0.08691(9)$ & $0.0252(7)$ \\
\hline $\mathrm{C} 16$ & $0.3952(2)$ & $0.37268(18)$ & $0.02826(9)$ & $0.0291(7)$ \\
\hline C17 & $0.6724(2)$ & $0.30665(17)$ & $0.03383(9)$ & $0.0270(7)$ \\
\hline $\mathrm{C} 18$ & $0.7864(2)$ & $0.24086(18)$ & $0.04111(9)$ & $0.0279(7)$ \\
\hline C19 & $0.9189(2)$ & $0.09679(19)$ & $0.16166(9)$ & $0.0278(7)$ \\
\hline $\mathrm{C} 20$ & $0.9764(2)$ & $0.0633(2)$ & $0.07007(10)$ & $0.0352(8)$ \\
\hline $\mathrm{C} 21$ & $0.7047(2)$ & $-0.06059(18)$ & $0.04720(8)$ & $0.0274(7)$ \\
\hline $\mathrm{C} 22$ & $0.5666(2)$ & $-0.08356(18)$ & $0.06159(8)$ & $0.0259(7)$ \\
\hline $\mathrm{C} 23$ & $0.5242(2)$ & $-0.20936(17)$ & $0.20181(9)$ & $0.0278(7)$ \\
\hline $\mathrm{C} 24$ & $0.3731(2)$ & $-0.21047(18)$ & $0.11673(9)$ & $0.0304(7)$ \\
\hline $\mathrm{C} 25$ & $0.2906(2)$ & $-0.00910(17)$ & $0.17232(9)$ & $0.0255(7)$ \\
\hline $\mathrm{C} 26$ & $0.2880(2)$ & $0.09026(17)$ & $0.20192(9)$ & $0.0257(7)$ \\
\hline $\mathrm{C} 27$ & $0.6151(2)$ & $0.20230(17)$ & $0.26588(9)$ & $0.0257(7)$ \\
\hline $\mathrm{C} 28$ & $0.3973(2)$ & $0.23431(19)$ & $0.29592(9)$ & $0.0323(8)$ \\
\hline $\mathrm{C} 29$ & $0.65598(19)$ & $0.41796(16)$ & $0.19249(8)$ & $0.0209(6)$ \\
\hline $\mathrm{C} 30$ & $0.74298(19)$ & $0.45928(16)$ & $0.15280(8)$ & $0.0210(6)$ \\
\hline $\mathrm{C} 31$ & $0.8608(2)$ & $0.40440(18)$ & $0.15117(9)$ & $0.0279(7)$ \\
\hline C32 & $0.9543(2)$ & $0.44442(19)$ & $0.12113(10)$ & $0.0336(7)$ \\
\hline $\mathrm{C} 33$ & $0.9312(2)$ & 0.54209 (19) & $0.09329(9)$ & $0.0312(7)$ \\
\hline C34 & $0.8160(2)$ & $0.59963(18)$ & $0.09487(9)$ & $0.0289(7)$ \\
\hline $\mathrm{C} 35$ & $0.7220(2)$ & $0.55801(16)$ & $0.12428(8)$ & $0.0247(7)$ \\
\hline H1 & 0.47190 & 0.39840 & 0.24610 & $0.0250^{*}$ \\
\hline $\mathrm{H} 2$ & 0.57810 & 0.04950 & 0.19770 & $0.0230^{*}$ \\
\hline $\mathrm{H} 3 \mathrm{~N}$ & $0.7345(19)$ & $-0.0690(17)$ & $0.1721(7)$ & $0.0250^{*}$ \\
\hline H4 & 0.62440 & 0.15290 & 0.12510 & $0.0230^{*}$ \\
\hline H6 & 0.63730 & -0.20130 & 0.11650 & $0.0270^{*}$ \\
\hline H7 & 0.85420 & -0.09910 & 0.10740 & $0.0270^{*}$ \\
\hline H12 & 0.50810 & 0.46530 & 0.11140 & $0.0260^{*}$ \\
\hline H13A & 0.34150 & 0.51710 & 0.15350 & $0.0330^{*}$ \\
\hline H13B & 0.26240 & 0.42900 & 0.11410 & $0.0330^{*}$ \\
\hline H14A & 0.27030 & 0.29460 & 0.17860 & $0.0310^{*}$ \\
\hline H14B & 0.26260 & 0.40360 & 0.21490 & $0.0310^{*}$ \\
\hline H15A & 0.40200 & 0.17280 & 0.11960 & $0.0380^{*}$ \\
\hline H15B & 0.36690 & 0.16100 & 0.05660 & $0.0380^{*}$ \\
\hline $\mathrm{H} 15 \mathrm{C}$ & 0.27900 & 0.23750 & 0.08980 & $0.0380^{*}$ \\
\hline H16A & 0.39670 & 0.32400 & -0.00270 & $0.0440^{*}$ \\
\hline H16B & 0.44580 & 0.44000 & 0.02340 & $0.0440^{*}$ \\
\hline $\mathrm{H} 16 \mathrm{C}$ & 0.30480 & 0.39310 & 0.03200 & $0.0440^{*}$ \\
\hline H17 & 0.65510 & 0.36450 & 0.00850 & $0.0320^{*}$ \\
\hline H18 & 0.85890 & 0.24660 & 0.02140 & $0.0330^{*}$ \\
\hline H19A & 0.96960 & 0.03230 & 0.17580 & $0.0420 *$ \\
\hline
\end{tabular}




$\begin{array}{lllll}\text { H19B } & 0.84730 & 0.11020 & 0.18310 & 0.0420^{*} \\ \text { H19C } & 0.97560 & 0.16230 & 0.16310 & 0.0420^{*} \\ \text { H20A } & 0.94180 & 0.04710 & 0.03320 & 0.0530^{*} \\ \text { H20B } & 1.03400 & 0.00270 & 0.08400 & 0.0530^{*} \\ \text { H20C } & 1.02580 & 0.13290 & 0.07150 & 0.0530^{*} \\ \text { H21A } & 0.70470 & 0.00480 & 0.02350 & 0.0330^{*} \\ \text { H21B } & 0.73840 & -0.12560 & 0.02920 & 0.0330^{*} \\ \text { H22A } & 0.51370 & -0.01480 & 0.06010 & 0.0310^{*} \\ \text { H22B } & 0.52110 & -0.13980 & 0.03760 & 0.0310^{*} \\ \text { H23A } & 0.45140 & -0.21860 & 0.22300 & 0.0420^{*} \\ \text { H23B } & 0.59530 & -0.16920 & 0.22280 & 0.0420^{*} \\ \text { H23C } & 0.55540 & -0.28250 & 0.19200 & 0.0420^{*} \\ \text { H24A } & 0.33490 & -0.16520 & 0.08670 & 0.0450^{*} \\ \text { H24B } & 0.30480 & -0.23240 & 0.13840 & 0.0450^{*} \\ \text { H24C } & 0.41300 & -0.27700 & 0.10330 & 0.0450^{*} \\ \text { H25 } & 0.21650 & -0.05050 & 0.15780 & 0.0310^{*} \\ \text { H26 } & 0.21210 & 0.12650 & 0.21120 & 0.0310^{*} \\ \text { H27A } & 0.64820 & 0.26010 & 0.29120 & 0.0380^{*} \\ \text { H27B } & 0.66550 & 0.20300 & 0.23540 & 0.0380^{*} \\ \text { H27C } & 0.62380 & 0.12960 & 0.28330 & 0.0380^{*} \\ \text { H28A } & 0.43610 & 0.29370 & 0.31910 & 0.0480^{*} \\ \text { H28B } & 0.30550 & 0.25160 & 0.28460 & 0.0480^{*} \\ \text { H28C } & 0.40330 & 0.16380 & 0.31540 & 0.0480^{*} \\ \text { H31 } & 0.87760 & 0.33800 & 0.17110 & 0.0330^{*} \\ \text { H32 } & 1.03350 & 0.40500 & 0.11970 & 0.0400^{*} \\ \text { H34 } & 0.80130 & 0.66740 & 0.07590 & 0.0350^{*} \\ \text { H35 } & 0.64230 & 0.59720 & 0.12500 & 0.0300^{*} \\ & & & & \end{array}$

Atomic displacement parameters $\left(\AA^{2}\right)$

\begin{tabular}{lllllll}
\hline & $U^{11}$ & $U^{22}$ & $U^{33}$ & $U^{12}$ & $U^{13}$ & $U^{23}$ \\
\hline C11 & $0.0417(4)$ & $0.0663(5)$ & $0.0499(4)$ & $-0.0137(3)$ & $0.0153(3)$ & $0.0165(4)$ \\
O1 & $0.0300(8)$ & $0.0350(9)$ & $0.0190(8)$ & $-0.0049(7)$ & $-0.0032(6)$ & $-0.0026(7)$ \\
N1 & $0.0205(9)$ & $0.0188(8)$ & $0.0168(9)$ & $0.0017(7)$ & $-0.0012(7)$ & $0.0010(7)$ \\
N2 & $0.0145(8)$ & $0.0212(8)$ & $0.0213(9)$ & $-0.0005(7)$ & $0.0023(7)$ & $0.0011(7)$ \\
N3 & $0.0221(9)$ & $0.0238(9)$ & $0.0150(9)$ & $0.0003(7)$ & $-0.0004(7)$ & $0.0019(7)$ \\
N4 & $0.0221(9)$ & $0.0218(9)$ & $0.0147(9)$ & $-0.0017(7)$ & $0.0019(7)$ & $0.0051(7)$ \\
C1 & $0.0199(10)$ & $0.0219(10)$ & $0.0217(11)$ & $0.0029(8)$ & $0.0035(8)$ & $-0.0018(9)$ \\
C2 & $0.0223(10)$ & $0.0223(10)$ & $0.0191(11)$ & $0.0030(8)$ & $0.0039(8)$ & $-0.0001(9)$ \\
C3 & $0.0211(10)$ & $0.0215(10)$ & $0.0183(11)$ & $0.0031(8)$ & $0.0046(8)$ & $0.0051(8)$ \\
C4 & $0.0221(11)$ & $0.0219(10)$ & $0.0180(11)$ & $-0.0027(8)$ & $0.0010(8)$ & $0.0045(9)$ \\
C5 & $0.0267(11)$ & $0.0180(10)$ & $0.0218(11)$ & $-0.0041(8)$ & $0.0012(9)$ & $-0.0005(9)$ \\
C6 & $0.0254(11)$ & $0.0178(10)$ & $0.0237(12)$ & $0.0016(8)$ & $0.0002(9)$ & $-0.0022(9)$ \\
C7 & $0.0237(11)$ & $0.0229(10)$ & $0.0211(11)$ & $0.0056(9)$ & $0.0050(9)$ & $-0.0009(9)$ \\
C8 & $0.0196(10)$ & $0.0283(11)$ & $0.0223(11)$ & $0.0005(9)$ & $0.0039(8)$ & $0.0011(9)$ \\
C9 & $0.0203(10)$ & $0.0230(10)$ & $0.0202(11)$ & $-0.0035(8)$ & $0.0029(8)$ & $-0.0005(9)$ \\
C10 & $0.0240(11)$ & $0.0196(10)$ & $0.0163(11)$ & $-0.0036(8)$ & $-0.0024(8)$ & $0.0000(8)$ \\
C11 & $0.0219(11)$ & $0.0226(10)$ & $0.0177(11)$ & $-0.0021(8)$ & $-0.0034(8)$ & $0.0032(9)$
\end{tabular}




$\begin{array}{lllllll}\mathrm{C} 12 & 0.0229(11) & 0.0190(10) & 0.0223(11) & 0.0000(8) & -0.0038(8) & 0.0051(9) \\ \text { C13 } & 0.0247(11) & 0.0231(11) & 0.0340(13) & 0.0049(9) & 0.0001(9) & 0.0020(10) \\ \text { C14 } & 0.0240(11) & 0.0245(11) & 0.0283(12) & 0.0065(9) & 0.0048(9) & 0.0025(9) \\ \text { C15 } & 0.0237(11) & 0.0263(11) & 0.0240(12) & -0.0035(9) & -0.0033(9) & 0.0011(9) \\ \text { C16 } & 0.0293(12) & 0.0318(12) & 0.0243(12) & 0.0007(10) & -0.0048(9) & 0.0064(10) \\ \text { C17 } & 0.0330(12) & 0.0270(12) & 0.0207(11) & -0.0028(9) & 0.0021(9) & 0.0059(9) \\ \text { C18 } & 0.0290(12) & 0.0335(12) & 0.0224(12) & -0.0042(10) & 0.0087(9) & 0.0031(10) \\ \text { C19 } & 0.0218(11) & 0.0307(12) & 0.0297(13) & 0.0003(9) & -0.0017(9) & 0.0026(10) \\ \text { C20 } & 0.0263(12) & 0.0431(14) & 0.0379(14) & 0.0040(10) & 0.0105(10) & 0.0044(11) \\ \text { C21 } & 0.0325(12) & 0.0279(12) & 0.0216(12) & -0.0034(9) & 0.0030(9) & -0.0039(9) \\ \text { C22 } & 0.0295(12) & 0.0254(11) & 0.0220(12) & -0.0016(9) & -0.0005(9) & -0.0029(9) \\ \text { C23 } & 0.0314(12) & 0.0230(11) & 0.0295(13) & 0.0012(9) & 0.0052(10) & 0.0036(10) \\ \text { C24 } & 0.0311(12) & 0.0295(11) & 0.0306(13) & -0.0083(10) & 0.0043(10) & -0.0036(10) \\ \text { C25 } & 0.0200(11) & 0.0250(11) & 0.0305(13) & -0.0047(9) & -0.0015(9) & 0.0072(10) \\ \text { C26 } & 0.0203(11) & 0.0252(11) & 0.0322(13) & 0.0024(9) & 0.0062(9) & 0.0067(10) \\ \text { C27 } & 0.0289(12) & 0.0209(10) & 0.0252(12) & 0.0027(9) & -0.0050(9) & -0.0001(9) \\ \text { C28 } & 0.0420(14) & 0.0317(12) & 0.0249(13) & 0.0069(10) & 0.0107(10) & 0.0024(10) \\ \text { C29 } & 0.0238(11) & 0.0158(10) & 0.0222(12) & 0.0029(8) & -0.0006(9) & -0.0015(9) \\ \text { C30 } & 0.0225(11) & 0.0204(10) & 0.0187(11) & -0.0049(8) & -0.0037(8) & -0.0045(9) \\ \text { C31 } & 0.0240(11) & 0.0252(11) & 0.0336(13) & 0.0000(9) & -0.0003(9) & 0.0033(10) \\ \text { C32 } & 0.0233(11) & 0.0354(13) & 0.0422(14) & 0.0033(10) & 0.0038(10) & 0.0017(11) \\ \text { C33 } & 0.0285(12) & 0.0400(13) & 0.0252(12) & -0.0121(10) & 0.0042(9) & 0.0000(11) \\ \text { C34 } & 0.0347(13) & 0.0263(11) & 0.0235(12) & -0.0074(10) & -0.0058(9) & 0.0037(10) \\ \text { C35 } & 0.0257(11) & 0.0217(11) & 0.0251(12) & 0.0006(9) & -0.0035(9) & -0.0015(9) \\ & & & & & & \end{array}$

Geometric parameters $\left(\AA,{ }^{\circ}\right)$

\begin{tabular}{llll}
\hline $\mathrm{C} 11-\mathrm{C} 33$ & $1.745(2)$ & $\mathrm{C} 33-\mathrm{C} 34$ & $1.381(3)$ \\
$\mathrm{O} 1-\mathrm{C} 29$ & $1.233(3)$ & $\mathrm{C} 34-\mathrm{C} 35$ & $1.385(3)$ \\
$\mathrm{N} 1-\mathrm{C} 1$ & $1.503(3)$ & $\mathrm{C} 1-\mathrm{H} 1$ & 1.0000 \\
$\mathrm{~N} 1-\mathrm{C} 12$ & $1.496(3)$ & $\mathrm{C} 6-\mathrm{H} 6$ & 1.0000 \\
$\mathrm{~N} 1-\mathrm{C} 29$ & $1.368(3)$ & $\mathrm{C} 7-\mathrm{H} 7$ & 1.0000 \\
$\mathrm{~N} 2-\mathrm{C} 3$ & $1.383(3)$ & $\mathrm{C} 12-\mathrm{H} 12$ & 1.0000 \\
$\mathrm{~N} 2-\mathrm{C} 4$ & $1.376(2)$ & $\mathrm{C} 13-\mathrm{H} 13 \mathrm{~A}$ & 0.9900 \\
$\mathrm{~N} 3-\mathrm{C} 6$ & $1.475(3)$ & $\mathrm{C} 13-\mathrm{H} 13 \mathrm{~B}$ & 0.9900 \\
$\mathrm{~N} 3-\mathrm{C} 7$ & $1.482(3)$ & $\mathrm{C} 14-\mathrm{H} 14 \mathrm{~A}$ & 0.9900 \\
$\mathrm{~N} 4-\mathrm{C} 9$ & $\mathrm{C} 14-\mathrm{H} 14 \mathrm{~B}$ & 0.9900 \\
$\mathrm{~N} 4-\mathrm{C} 10$ & $1.380(3)$ & $\mathrm{C} 15-\mathrm{H} 15 \mathrm{~A}$ & 0.9800 \\
$\mathrm{~N} 2-\mathrm{H} 2$ & $1.378(3)$ & $\mathrm{C} 15-\mathrm{H} 15 \mathrm{~B}$ & 0.9800 \\
$\mathrm{~N} 3-\mathrm{H} 3 \mathrm{~N}$ & 0.8800 & $\mathrm{C} 15-\mathrm{H} 15 \mathrm{C}$ & 0.9800 \\
$\mathrm{~N} 4-\mathrm{H} 4$ & $0.882(18)$ & $\mathrm{C} 16-\mathrm{H} 16 \mathrm{~A}$ & 0.9800 \\
$\mathrm{C} 1-\mathrm{C} 2$ & 0.8800 & $\mathrm{C} 16-\mathrm{H} 16 \mathrm{~B}$ & 0.9800 \\
$\mathrm{C} 1-\mathrm{C} 14$ & $1.576(3)$ & $\mathrm{C} 16-\mathrm{H} 16 \mathrm{C}$ & 0.9800 \\
$\mathrm{C} 2-\mathrm{C} 3$ & $1.536(3)$ & $\mathrm{C} 17-\mathrm{H} 17$ & 0.9500 \\
$\mathrm{C} 2-\mathrm{C} 27$ & $1.518(3)$ & $\mathrm{C} 18-\mathrm{H} 18$ & 0.9500 \\
$\mathrm{C} 2-\mathrm{C} 28$ & $1.530(3)$ & $\mathrm{C} 19-\mathrm{H} 19 \mathrm{~A}$ & 0.9800 \\
$\mathrm{C} 3-\mathrm{C} 26$ & $1.543(3)$ & $\mathrm{C} 19-\mathrm{H} 19 \mathrm{~B}$ & 0.9800 \\
$\mathrm{C} 4-\mathrm{C} 5$ & $1.373(3)$ & $\mathrm{C} 19-\mathrm{H} 19 \mathrm{C}$ & 0.9800
\end{tabular}




\begin{tabular}{|c|c|c|c|}
\hline $\mathrm{C} 4-\mathrm{C} 25$ & $1.370(3)$ & $\mathrm{C} 20-\mathrm{H} 20 \mathrm{~A}$ & 0.9800 \\
\hline $\mathrm{C} 5-\mathrm{C} 23$ & $1.546(3)$ & $\mathrm{C} 20-\mathrm{H} 20 \mathrm{~B}$ & 0.9800 \\
\hline $\mathrm{C} 5-\mathrm{C} 6$ & $1.545(3)$ & $\mathrm{C} 20-\mathrm{H} 20 \mathrm{C}$ & 0.9800 \\
\hline $\mathrm{C} 5-\mathrm{C} 24$ & $1.538(3)$ & $\mathrm{C} 21-\mathrm{H} 21 \mathrm{~A}$ & 0.9900 \\
\hline $\mathrm{C} 6-\mathrm{C} 22$ & $1.527(3)$ & $\mathrm{C} 21-\mathrm{H} 21 \mathrm{~B}$ & 0.9900 \\
\hline $\mathrm{C} 7-\mathrm{C} 8$ & $1.552(3)$ & $\mathrm{C} 22-\mathrm{H} 22 \mathrm{~A}$ & 0.9900 \\
\hline $\mathrm{C} 7-\mathrm{C} 21$ & $1.549(3)$ & $\mathrm{C} 22-\mathrm{H} 22 \mathrm{~B}$ & 0.9900 \\
\hline $\mathrm{C} 8-\mathrm{C} 20$ & $1.538(3)$ & $\mathrm{C} 23-\mathrm{H} 23 \mathrm{~A}$ & 0.9800 \\
\hline $\mathrm{C} 8-\mathrm{C} 9$ & $1.512(3)$ & $\mathrm{C} 23-\mathrm{H} 23 \mathrm{~B}$ & 0.9800 \\
\hline $\mathrm{C} 8-\mathrm{C} 19$ & $1.532(3)$ & $\mathrm{C} 23-\mathrm{H} 23 \mathrm{C}$ & 0.9800 \\
\hline $\mathrm{C} 9-\mathrm{C} 18$ & $1.369(3)$ & $\mathrm{C} 24-\mathrm{H} 24 \mathrm{~A}$ & 0.9800 \\
\hline $\mathrm{C} 10-\mathrm{C} 11$ & $1.520(3)$ & $\mathrm{C} 24-\mathrm{H} 24 \mathrm{~B}$ & 0.9800 \\
\hline $\mathrm{C} 10-\mathrm{C} 17$ & $1.373(3)$ & $\mathrm{C} 24-\mathrm{H} 24 \mathrm{C}$ & 0.9800 \\
\hline $\mathrm{C} 11-\mathrm{C} 16$ & $1.539(3)$ & $\mathrm{C} 25-\mathrm{H} 25$ & 0.9500 \\
\hline $\mathrm{C} 11-\mathrm{C} 12$ & $1.555(3)$ & $\mathrm{C} 26-\mathrm{H} 26$ & 0.9500 \\
\hline $\mathrm{C} 11-\mathrm{C} 15$ & $1.529(3)$ & $\mathrm{C} 27-\mathrm{H} 27 \mathrm{~A}$ & 0.9800 \\
\hline $\mathrm{C} 12-\mathrm{C} 13$ & $1.539(3)$ & $\mathrm{C} 27-\mathrm{H} 27 \mathrm{~B}$ & 0.9800 \\
\hline $\mathrm{C} 13-\mathrm{C} 14$ & $1.530(3)$ & $\mathrm{C} 27-\mathrm{H} 27 \mathrm{C}$ & 0.9800 \\
\hline $\mathrm{C} 17-\mathrm{C} 18$ & $1.412(3)$ & $\mathrm{C} 28-\mathrm{H} 28 \mathrm{~A}$ & 0.9800 \\
\hline $\mathrm{C} 21-\mathrm{C} 22$ & $1.538(3)$ & $\mathrm{C} 28-\mathrm{H} 28 \mathrm{~B}$ & 0.9800 \\
\hline $\mathrm{C} 25-\mathrm{C} 26$ & $1.414(3)$ & $\mathrm{C} 28-\mathrm{H} 28 \mathrm{C}$ & 0.9800 \\
\hline $\mathrm{C} 29-\mathrm{C} 30$ & $1.510(3)$ & $\mathrm{C} 31-\mathrm{H} 31$ & 0.9500 \\
\hline $\mathrm{C} 30-\mathrm{C} 35$ & $1.395(3)$ & $\mathrm{C} 32-\mathrm{H} 32$ & 0.9500 \\
\hline $\mathrm{C} 30-\mathrm{C} 31$ & $1.389(3)$ & $\mathrm{C} 34-\mathrm{H} 34$ & 0.9500 \\
\hline $\mathrm{C} 31-\mathrm{C} 32$ & $1.384(3)$ & $\mathrm{C} 35-\mathrm{H} 35$ & 0.9500 \\
\hline $\mathrm{C} 32-\mathrm{C} 33$ & $1.377(3)$ & & \\
\hline $\mathrm{C} 1-\mathrm{N} 1-\mathrm{C} 12$ & $111.93(15)$ & $\mathrm{C} 21-\mathrm{C} 7-\mathrm{H} 7$ & 108.00 \\
\hline $\mathrm{C} 1-\mathrm{N} 1-\mathrm{C} 29$ & $116.78(15)$ & $\mathrm{N} 1-\mathrm{C} 12-\mathrm{H} 12$ & 107.00 \\
\hline $\mathrm{C} 12-\mathrm{N} 1-\mathrm{C} 29$ & $119.69(15)$ & $\mathrm{C} 11-\mathrm{C} 12-\mathrm{H} 12$ & 107.00 \\
\hline $\mathrm{C} 3-\mathrm{N} 2-\mathrm{C} 4$ & $110.96(16)$ & $\mathrm{C} 13-\mathrm{C} 12-\mathrm{H} 12$ & 107.00 \\
\hline $\mathrm{C} 6-\mathrm{N} 3-\mathrm{C} 7$ & $106.09(15)$ & $\mathrm{C} 12-\mathrm{C} 13-\mathrm{H} 13 \mathrm{~A}$ & 111.00 \\
\hline $\mathrm{C} 9-\mathrm{N} 4-\mathrm{C} 10$ & $110.73(16)$ & $\mathrm{C} 12-\mathrm{C} 13-\mathrm{H} 13 \mathrm{~B}$ & 111.00 \\
\hline $\mathrm{C} 4-\mathrm{N} 2-\mathrm{H} 2$ & 125.00 & $\mathrm{C} 14-\mathrm{C} 13-\mathrm{H} 13 \mathrm{~A}$ & 111.00 \\
\hline $\mathrm{C} 3-\mathrm{N} 2-\mathrm{H} 2$ & 124.00 & $\mathrm{C} 14-\mathrm{C} 13-\mathrm{H} 13 \mathrm{~B}$ & 111.00 \\
\hline $\mathrm{C} 6-\mathrm{N} 3-\mathrm{H} 3 \mathrm{~N}$ & $113.1(13)$ & $\mathrm{H} 13 \mathrm{~A}-\mathrm{C} 13-\mathrm{H} 13 \mathrm{~B}$ & 109.00 \\
\hline $\mathrm{C} 7-\mathrm{N} 3-\mathrm{H} 3 \mathrm{~N}$ & $111.6(13)$ & $\mathrm{C} 1-\mathrm{C} 14-\mathrm{H} 14 \mathrm{~A}$ & 111.00 \\
\hline $\mathrm{C} 10-\mathrm{N} 4-\mathrm{H} 4$ & 125.00 & $\mathrm{C} 1-\mathrm{C} 14-\mathrm{H} 14 \mathrm{~B}$ & 111.00 \\
\hline $\mathrm{C} 9-\mathrm{N} 4-\mathrm{H} 4$ & 125.00 & $\mathrm{C} 13-\mathrm{C} 14-\mathrm{H} 14 \mathrm{~A}$ & 111.00 \\
\hline $\mathrm{N} 1-\mathrm{C} 1-\mathrm{C} 14$ & $104.17(16)$ & $\mathrm{C} 13-\mathrm{C} 14-\mathrm{H} 14 \mathrm{~B}$ & 111.00 \\
\hline $\mathrm{N} 1-\mathrm{C} 1-\mathrm{C} 2$ & $117.18(16)$ & $\mathrm{H} 14 \mathrm{~A}-\mathrm{C} 14-\mathrm{H} 14 \mathrm{~B}$ & 109.00 \\
\hline $\mathrm{C} 2-\mathrm{C} 1-\mathrm{C} 14$ & $115.36(16)$ & $\mathrm{C} 11-\mathrm{C} 15-\mathrm{H} 15 \mathrm{~A}$ & 109.00 \\
\hline $\mathrm{C} 1-\mathrm{C} 2-\mathrm{C} 28$ & $105.28(16)$ & $\mathrm{C} 11-\mathrm{C} 15-\mathrm{H} 15 \mathrm{~B}$ & 109.00 \\
\hline $\mathrm{C} 1-\mathrm{C} 2-\mathrm{C} 3$ & $115.78(16)$ & $\mathrm{C} 11-\mathrm{C} 15-\mathrm{H} 15 \mathrm{C}$ & 109.00 \\
\hline $\mathrm{C} 1-\mathrm{C} 2-\mathrm{C} 27$ & $111.11(16)$ & $\mathrm{H} 15 \mathrm{~A}-\mathrm{C} 15-\mathrm{H} 15 \mathrm{~B}$ & 110.00 \\
\hline $\mathrm{C} 27-\mathrm{C} 2-\mathrm{C} 28$ & $108.11(17)$ & $\mathrm{H} 15 \mathrm{~A}-\mathrm{C} 15-\mathrm{H} 15 \mathrm{C}$ & 109.00 \\
\hline $\mathrm{C} 3-\mathrm{C} 2-\mathrm{C} 27$ & $109.37(16)$ & $\mathrm{H} 15 \mathrm{~B}-\mathrm{C} 15-\mathrm{H} 15 \mathrm{C}$ & 109.00 \\
\hline $\mathrm{C} 3-\mathrm{C} 2-\mathrm{C} 28$ & $106.78(16)$ & $\mathrm{C} 11-\mathrm{C} 16-\mathrm{H} 16 \mathrm{~A}$ & 109.00 \\
\hline
\end{tabular}




\begin{tabular}{|c|c|}
\hline $\mathrm{C} 2-\mathrm{C} 3-\mathrm{C} 26$ & $131.91(18)$ \\
\hline $\mathrm{N} 2-\mathrm{C} 3-\mathrm{C} 26$ & $106.15(17)$ \\
\hline $\mathrm{N} 2-\mathrm{C} 3-\mathrm{C} 2$ & $121.91(17)$ \\
\hline $\mathrm{C} 5-\mathrm{C} 4-\mathrm{C} 25$ & $130.28(18)$ \\
\hline $\mathrm{N} 2-\mathrm{C} 4-\mathrm{C} 5$ & $122.03(17)$ \\
\hline $\mathrm{N} 2-\mathrm{C} 4-\mathrm{C} 25$ & $106.35(17)$ \\
\hline $\mathrm{C} 4-\mathrm{C} 5-\mathrm{C} 6$ & $114.84(16)$ \\
\hline $\mathrm{C} 6-\mathrm{C} 5-\mathrm{C} 23$ & $108.88(16)$ \\
\hline $\mathrm{C} 4-\mathrm{C} 5-\mathrm{C} 23$ & $107.28(16)$ \\
\hline $\mathrm{C} 4-\mathrm{C} 5-\mathrm{C} 24$ & $109.32(17)$ \\
\hline $\mathrm{C} 6-\mathrm{C} 5-\mathrm{C} 24$ & $107.66(16)$ \\
\hline $\mathrm{C} 23-\mathrm{C} 5-\mathrm{C} 24$ & $108.73(16)$ \\
\hline $\mathrm{N} 3-\mathrm{C} 6-\mathrm{C} 22$ & $100.71(16)$ \\
\hline $\mathrm{N} 3-\mathrm{C} 6-\mathrm{C} 5$ & $115.48(16)$ \\
\hline $\mathrm{C} 5-\mathrm{C} 6-\mathrm{C} 22$ & $118.22(17)$ \\
\hline $\mathrm{N} 3-\mathrm{C} 7-\mathrm{C} 8$ & $113.32(16)$ \\
\hline $\mathrm{N} 3-\mathrm{C} 7-\mathrm{C} 21$ & $104.13(16)$ \\
\hline $\mathrm{C} 8-\mathrm{C} 7-\mathrm{C} 21$ & $114.65(17)$ \\
\hline $\mathrm{C} 7-\mathrm{C} 8-\mathrm{C} 20$ & $107.59(17)$ \\
\hline $\mathrm{C} 9-\mathrm{C} 8-\mathrm{C} 19$ & $111.74(17)$ \\
\hline $\mathrm{C} 7-\mathrm{C} 8-\mathrm{C} 9$ & $110.86(16)$ \\
\hline $\mathrm{C} 7-\mathrm{C} 8-\mathrm{C} 19$ & 109.21 \\
\hline $\mathrm{C} 9-\mathrm{C} 8-\mathrm{C} 20$ & $108.63(17)$ \\
\hline $\mathrm{C} 19-\mathrm{C} 8-\mathrm{C} 20$ & 108.70 \\
\hline $\mathrm{N} 4-\mathrm{C} 9-\mathrm{C} 8$ & $122.41(17)$ \\
\hline $\mathrm{N} 4-\mathrm{C} 9-\mathrm{C} 18$ & $106.47(17)$ \\
\hline $\mathrm{C} 8-\mathrm{C} 9-\mathrm{C} 18$ & $131.03(18)$ \\
\hline $\mathrm{N} 4-\mathrm{C} 10-\mathrm{C} 17$ & $106.38(17)$ \\
\hline $\mathrm{N} 4-\mathrm{C} 10-\mathrm{C} 11$ & $122.76(17)$ \\
\hline $\mathrm{C} 11-\mathrm{C} 10-\mathrm{C} 17$ & 130.85 \\
\hline $\mathrm{C} 12-\mathrm{C} 11-\mathrm{C} 16$ & $106.18(16)$ \\
\hline $\mathrm{C} 10-\mathrm{C} 11-\mathrm{C} 15$ & $110.31(16)$ \\
\hline $\mathrm{C} 15-\mathrm{C} 11-\mathrm{C} 16$ & $107.75(17)$ \\
\hline $\mathrm{C} 10-\mathrm{C} 11-\mathrm{C} 12$ & $109.88(16)$ \\
\hline $\mathrm{C} 10-\mathrm{C} 11-\mathrm{C} 16$ & $108.21(16)$ \\
\hline $\mathrm{C} 12-\mathrm{C} 11-\mathrm{C} 15$ & $114.24(17)$ \\
\hline $\mathrm{N} 1-\mathrm{C} 12-\mathrm{C} 13$ & $101.44(16)$ \\
\hline $\mathrm{N} 1-\mathrm{C} 12-\mathrm{C} 11$ & $117.13(16)$ \\
\hline $\mathrm{C} 11-\mathrm{C} 12-\mathrm{C} 13$ & $117.19(17)$ \\
\hline $\mathrm{C} 12-\mathrm{C} 13-\mathrm{C} 14$ & $105.32(16)$ \\
\hline $\mathrm{C} 1-\mathrm{C} 14-\mathrm{C} 13$ & $104.98(16)$ \\
\hline $\mathrm{C} 10-\mathrm{C} 17-\mathrm{C} 18$ & $108.17(19)$ \\
\hline $\mathrm{C} 9-\mathrm{C} 18-\mathrm{C} 17$ & $108.24(19)$ \\
\hline $\mathrm{C} 7-\mathrm{C} 21-\mathrm{C} 22$ & $105.11(16)$ \\
\hline $\mathrm{C} 6-\mathrm{C} 22-\mathrm{C} 21$ & $102.14(16)$ \\
\hline $\mathrm{C} 4-\mathrm{C} 25-\mathrm{C} 26$ & 108.38 \\
\hline $\mathrm{C} 3-\mathrm{C} 26-\mathrm{C} 25$ & $108.16(18)$ \\
\hline $\mathrm{O} 1-\mathrm{C} 29-\mathrm{N} 1$ & $122.32(18)$ \\
\hline
\end{tabular}

\begin{tabular}{|c|c|}
\hline $\mathrm{C} 11-\mathrm{C} 16-\mathrm{H} 16 \mathrm{~B}$ & 109.00 \\
\hline $\mathrm{C} 11-\mathrm{C} 16-\mathrm{H} 16 \mathrm{C}$ & 110.00 \\
\hline $\mathrm{H} 16 \mathrm{~A}-\mathrm{C} 16-\mathrm{H} 16 \mathrm{~B}$ & 110.00 \\
\hline $\mathrm{H} 16 \mathrm{~A}-\mathrm{C} 16-\mathrm{H} 16 \mathrm{C}$ & 109.00 \\
\hline $\mathrm{H} 16 \mathrm{~B}-\mathrm{C} 16-\mathrm{H} 16 \mathrm{C}$ & 109.00 \\
\hline $\mathrm{C} 10-\mathrm{C} 17-\mathrm{H} 17$ & 126.00 \\
\hline $\mathrm{C} 18-\mathrm{C} 17-\mathrm{H} 17$ & 126.00 \\
\hline $\mathrm{C} 9-\mathrm{C} 18-\mathrm{H} 18$ & 126.00 \\
\hline $\mathrm{C} 17-\mathrm{C} 18-\mathrm{H} 18$ & 126.00 \\
\hline $\mathrm{C} 8-\mathrm{C} 19-\mathrm{H} 19 \mathrm{~A}$ & 110.00 \\
\hline $\mathrm{C} 8-\mathrm{C} 19-\mathrm{H} 19 \mathrm{~B}$ & 109.00 \\
\hline $\mathrm{C} 8-\mathrm{C} 19-\mathrm{H} 19 \mathrm{C}$ & 110.00 \\
\hline $\mathrm{H} 19 \mathrm{~A}-\mathrm{C} 19-\mathrm{H} 19 \mathrm{~B}$ & 109.00 \\
\hline $\mathrm{H} 19 \mathrm{~A}-\mathrm{C} 19-\mathrm{H} 19 \mathrm{C}$ & 109.00 \\
\hline $\mathrm{H} 19 \mathrm{~B}-\mathrm{C} 19-\mathrm{H} 19 \mathrm{C}$ & 109.00 \\
\hline $\mathrm{C} 8-\mathrm{C} 20-\mathrm{H} 20 \mathrm{~A}$ & 109.00 \\
\hline $\mathrm{C} 8-\mathrm{C} 20-\mathrm{H} 20 \mathrm{~B}$ & 109.00 \\
\hline $\mathrm{C} 8-\mathrm{C} 20-\mathrm{H} 20 \mathrm{C}$ & 109.00 \\
\hline $\mathrm{H} 20 \mathrm{~A}-\mathrm{C} 20-\mathrm{H} 20 \mathrm{~B}$ & 109.00 \\
\hline $\mathrm{H} 20 \mathrm{~A}-\mathrm{C} 20-\mathrm{H} 20 \mathrm{C}$ & 110.00 \\
\hline $\mathrm{H} 20 \mathrm{~B}-\mathrm{C} 20-\mathrm{H} 20 \mathrm{C}$ & 109.00 \\
\hline $\mathrm{C} 7-\mathrm{C} 21-\mathrm{H} 21 \mathrm{~A}$ & 111.00 \\
\hline $\mathrm{C} 7-\mathrm{C} 21-\mathrm{H} 21 \mathrm{~B}$ & 111.00 \\
\hline $\mathrm{C} 22-\mathrm{C} 21-\mathrm{H} 21 \mathrm{~A}$ & 111.00 \\
\hline $\mathrm{C} 22-\mathrm{C} 21-\mathrm{H} 21 \mathrm{~B}$ & 111.00 \\
\hline $\mathrm{H} 21 \mathrm{~A}-\mathrm{C} 21-\mathrm{H} 21 \mathrm{~B}$ & 109.00 \\
\hline $\mathrm{C} 6-\mathrm{C} 22-\mathrm{H} 22 \mathrm{~A}$ & 111.00 \\
\hline $\mathrm{C} 6-\mathrm{C} 22-\mathrm{H} 22 \mathrm{~B}$ & 111.00 \\
\hline $\mathrm{C} 21-\mathrm{C} 22-\mathrm{H} 22 \mathrm{~A}$ & 111.00 \\
\hline $\mathrm{C} 21-\mathrm{C} 22-\mathrm{H} 22 \mathrm{~B}$ & 111.00 \\
\hline $\mathrm{H} 22 \mathrm{~A}-\mathrm{C} 22-\mathrm{H} 22 \mathrm{~B}$ & 109.00 \\
\hline $\mathrm{C} 5-\mathrm{C} 23-\mathrm{H} 23 \mathrm{~A}$ & 110.00 \\
\hline $\mathrm{C} 5-\mathrm{C} 23-\mathrm{H} 23 \mathrm{~B}$ & 109.00 \\
\hline $\mathrm{C} 5-\mathrm{C} 23-\mathrm{H} 23 \mathrm{C}$ & 109.00 \\
\hline $\mathrm{H} 23 \mathrm{~A}-\mathrm{C} 23-\mathrm{H} 23 \mathrm{~B}$ & 109.00 \\
\hline $\mathrm{H} 23 \mathrm{~A}-\mathrm{C} 23-\mathrm{H} 23 \mathrm{C}$ & 109.00 \\
\hline $\mathrm{H} 23 \mathrm{~B}-\mathrm{C} 23-\mathrm{H} 23 \mathrm{C}$ & 109.00 \\
\hline $\mathrm{C} 5-\mathrm{C} 24-\mathrm{H} 24 \mathrm{~A}$ & 109.00 \\
\hline $\mathrm{C} 5-\mathrm{C} 24-\mathrm{H} 24 \mathrm{~B}$ & 109.00 \\
\hline $\mathrm{C} 5-\mathrm{C} 24-\mathrm{H} 24 \mathrm{C}$ & 109.00 \\
\hline $\mathrm{H} 24 \mathrm{~A}-\mathrm{C} 24-\mathrm{H} 24 \mathrm{~B}$ & 110.00 \\
\hline $\mathrm{H} 24 \mathrm{~A}-\mathrm{C} 24-\mathrm{H} 24 \mathrm{C}$ & 109.00 \\
\hline $\mathrm{H} 24 \mathrm{~B}-\mathrm{C} 24-\mathrm{H} 24 \mathrm{C}$ & 110.00 \\
\hline $\mathrm{C} 4-\mathrm{C} 25-\mathrm{H} 25$ & 126.00 \\
\hline $\mathrm{C} 26-\mathrm{C} 25-\mathrm{H} 25$ & 126.00 \\
\hline $\mathrm{C} 3-\mathrm{C} 26-\mathrm{H} 26$ & 126.00 \\
\hline $\mathrm{C} 25-\mathrm{C} 26-\mathrm{H} 26$ & 126.00 \\
\hline $\mathrm{C} 2-\mathrm{C} 27-\mathrm{H} 27 \mathrm{~A}$ & 109.00 \\
\hline
\end{tabular}




\begin{tabular}{|c|c|}
\hline $\mathrm{N} 1-\mathrm{C} 29-\mathrm{C} 30$ & $120.88(17)$ \\
\hline $\mathrm{O} 1-\mathrm{C} 29-\mathrm{C} 30$ & $116.71(17)$ \\
\hline $\mathrm{C} 31-\mathrm{C} 30-\mathrm{C} 35$ & $118.22(19)$ \\
\hline $\mathrm{C} 29-\mathrm{C} 30-\mathrm{C} 35$ & $123.90(18)$ \\
\hline $\mathrm{C} 29-\mathrm{C} 30-\mathrm{C} 31$ & $117.07(18)$ \\
\hline $\mathrm{C} 30-\mathrm{C} 31-\mathrm{C} 32$ & $121.4(2)$ \\
\hline $\mathrm{C} 31-\mathrm{C} 32-\mathrm{C} 33$ & $119.3(2)$ \\
\hline $\mathrm{C} 11-\mathrm{C} 33-\mathrm{C} 32$ & $119.82(17)$ \\
\hline $\mathrm{C} 11-\mathrm{C} 33-\mathrm{C} 34$ & $119.44(17)$ \\
\hline $\mathrm{C} 32-\mathrm{C} 33-\mathrm{C} 34$ & $120.7(2)$ \\
\hline $\mathrm{C} 33-\mathrm{C} 34-\mathrm{C} 35$ & $119.6(2)$ \\
\hline $\mathrm{C} 30-\mathrm{C} 35-\mathrm{C} 34$ & $120.75(19)$ \\
\hline $\mathrm{N} 1-\mathrm{C} 1-\mathrm{H} 1$ & 106.00 \\
\hline $\mathrm{C} 2-\mathrm{C} 1-\mathrm{H} 1$ & 106.00 \\
\hline $\mathrm{C} 14-\mathrm{C} 1-\mathrm{H} 1$ & 106.00 \\
\hline $\mathrm{N} 3-\mathrm{C} 6-\mathrm{H} 6$ & 107.00 \\
\hline $\mathrm{C} 5-\mathrm{C} 6-\mathrm{H} 6$ & 107.00 \\
\hline $\mathrm{C} 22-\mathrm{C} 6-\mathrm{H} 6$ & 107.00 \\
\hline $\mathrm{N} 3-\mathrm{C} 7-\mathrm{H} 7$ & 108.00 \\
\hline $\mathrm{C} 8-\mathrm{C} 7-\mathrm{H} 7$ & 108.00 \\
\hline $\mathrm{C} 12-\mathrm{N} 1-\mathrm{C} 1-\mathrm{C} 2$ & $126.13(17)$ \\
\hline $\mathrm{C} 12-\mathrm{N} 1-\mathrm{C} 1-\mathrm{C} 14$ & $-2.6(2)$ \\
\hline $\mathrm{C} 29-\mathrm{N} 1-\mathrm{C} 1-\mathrm{C} 2$ & $-90.6(2)$ \\
\hline $\mathrm{C} 29-\mathrm{N} 1-\mathrm{C} 1-\mathrm{C} 14$ & $140.62(17)$ \\
\hline $\mathrm{C} 1-\mathrm{N} 1-\mathrm{C} 12-\mathrm{C} 11$ & $-106.27(18)$ \\
\hline $\mathrm{C} 1-\mathrm{N} 1-\mathrm{C} 12-\mathrm{C} 13$ & $22.59(19)$ \\
\hline $\mathrm{C} 29-\mathrm{N} 1-\mathrm{C} 12-\mathrm{C} 11$ & $111.7(2)$ \\
\hline $\mathrm{C} 29-\mathrm{N} 1-\mathrm{C} 12-\mathrm{C} 13$ & $-119.47(18)$ \\
\hline $\mathrm{C} 1-\mathrm{N} 1-\mathrm{C} 29-\mathrm{O} 1$ & $11.0(3)$ \\
\hline $\mathrm{C} 1-\mathrm{N} 1-\mathrm{C} 29-\mathrm{C} 30$ & $-172.54(16)$ \\
\hline $\mathrm{C} 12-\mathrm{N} 1-\mathrm{C} 29-\mathrm{O} 1$ & $151.33(18)$ \\
\hline $\mathrm{C} 12-\mathrm{N} 1-\mathrm{C} 29-\mathrm{C} 30$ & $-32.3(3)$ \\
\hline $\mathrm{C} 4-\mathrm{N} 2-\mathrm{C} 3-\mathrm{C} 2$ & $178.98(17)$ \\
\hline $\mathrm{C} 4-\mathrm{N} 2-\mathrm{C} 3-\mathrm{C} 26$ & $0.8(2)$ \\
\hline $\mathrm{C} 3-\mathrm{N} 2-\mathrm{C} 4-\mathrm{C} 5$ & $-168.17(18)$ \\
\hline $\mathrm{C} 3-\mathrm{N} 2-\mathrm{C} 4-\mathrm{C} 25$ & $-0.2(2)$ \\
\hline $\mathrm{C} 7-\mathrm{N} 3-\mathrm{C} 6-\mathrm{C} 5$ & $-174.32(16)$ \\
\hline $\mathrm{C} 7-\mathrm{N} 3-\mathrm{C} 6-\mathrm{C} 22$ & $-45.77(18)$ \\
\hline $\mathrm{C} 6-\mathrm{N} 3-\mathrm{C} 7-\mathrm{C} 8$ & $154.10(16)$ \\
\hline $\mathrm{C} 6-\mathrm{N} 3-\mathrm{C} 7-\mathrm{C} 21$ & $28.87(19)$ \\
\hline $\mathrm{C} 10-\mathrm{N} 4-\mathrm{C} 9-\mathrm{C} 8$ & $-176.00(18)$ \\
\hline $\mathrm{C} 10-\mathrm{N} 4-\mathrm{C} 9-\mathrm{C} 18$ & $0.9(2)$ \\
\hline $\mathrm{C} 9-\mathrm{N} 4-\mathrm{C} 10-\mathrm{C} 11$ & $177.92(17)$ \\
\hline $\mathrm{C} 9-\mathrm{N} 4-\mathrm{C} 10-\mathrm{C} 17$ & $-1.1(2)$ \\
\hline $\mathrm{N} 1-\mathrm{C} 1-\mathrm{C} 2-\mathrm{C} 3$ & $-76.6(2)$ \\
\hline $\mathrm{N} 1-\mathrm{C} 1-\mathrm{C} 2-\mathrm{C} 27$ & $48.9(2)$ \\
\hline $\mathrm{N} 1-\mathrm{C} 1-\mathrm{C} 2-\mathrm{C} 28$ & $165.73(16)$ \\
\hline
\end{tabular}

$\begin{array}{ll}\mathrm{C} 2-\mathrm{C} 27-\mathrm{H} 27 \mathrm{~B} & 109.00 \\ \mathrm{C} 2-\mathrm{C} 27-\mathrm{H} 27 \mathrm{C} & 109.00 \\ \mathrm{H} 27 \mathrm{~A}-\mathrm{C} 27-\mathrm{H} 27 \mathrm{~B} & 109.00 \\ \mathrm{H} 27 \mathrm{~A}-\mathrm{C} 27-\mathrm{H} 27 \mathrm{C} & 109.00 \\ \mathrm{H} 27 \mathrm{~B}-\mathrm{C} 27-\mathrm{H} 27 \mathrm{C} & 109.00 \\ \mathrm{C} 2-\mathrm{C} 28-\mathrm{H} 28 \mathrm{~A} & 109.00 \\ \mathrm{C} 2-\mathrm{C} 28-\mathrm{H} 28 \mathrm{~B} & 110.00 \\ \mathrm{C} 2-\mathrm{C} 28-\mathrm{H} 28 \mathrm{C} & 109.00 \\ \mathrm{H} 28 \mathrm{~A}-\mathrm{C} 28-\mathrm{H} 28 \mathrm{~B} & 109.00 \\ \mathrm{H} 28 \mathrm{~A}-\mathrm{C} 28-\mathrm{H} 28 \mathrm{C} & 109.00 \\ \mathrm{H} 28 \mathrm{~B}-\mathrm{C} 28-\mathrm{H} 28 \mathrm{C} & 109.00 \\ \mathrm{C} 30-\mathrm{C} 31-\mathrm{H} 31 & 119.00 \\ \mathrm{C} 32-\mathrm{C} 31-\mathrm{H} 31 & 119.00 \\ \mathrm{C} 31-\mathrm{C} 32-\mathrm{H} 32 & 120.00 \\ \mathrm{C} 33-\mathrm{C} 32-\mathrm{H} 32 & 120.00 \\ \mathrm{C} 33-\mathrm{C} 34-\mathrm{H} 34 & 120.00 \\ \mathrm{C} 35-\mathrm{C} 34-\mathrm{H} 34 & 120.00 \\ \mathrm{C} 30-\mathrm{C} 35-\mathrm{H} 35 & 120.00 \\ \mathrm{C} 34-\mathrm{C} 35-\mathrm{H} 35 & 120.00\end{array}$

$\mathrm{C} 24-\mathrm{C} 5-\mathrm{C} 6-\mathrm{C} 22 \quad 50.7$ (2)

$\mathrm{N} 3-\mathrm{C} 6-\mathrm{C} 22-\mathrm{C} 21$

$\mathrm{C} 5-\mathrm{C} 6-\mathrm{C} 22-\mathrm{C} 21 \quad 170.30(17)$

$\mathrm{N} 3-\mathrm{C} 7-\mathrm{C} 8-\mathrm{C} 9 \quad-74.1$ (2)

$\mathrm{N} 3-\mathrm{C} 7-\mathrm{C} 8-\mathrm{C} 19 \quad 49.4$ (2)

N3-C7-C8-C20 167.26 (17)

$\mathrm{C} 21-\mathrm{C} 7-\mathrm{C} 8-\mathrm{C} 9 \quad 45.3$ (2)

$\mathrm{C} 21-\mathrm{C} 7-\mathrm{C} 8-\mathrm{C} 19 \quad 168.80(17)$

$\mathrm{C} 21-\mathrm{C} 7-\mathrm{C} 8-\mathrm{C} 20 \quad-73.4$ (2)

$\mathrm{N} 3-\mathrm{C} 7-\mathrm{C} 21-\mathrm{C} 22 \quad-0.6(2)$

$\mathrm{C} 8-\mathrm{C} 7-\mathrm{C} 21-\mathrm{C} 22 \quad-124.95$ (18)

$\mathrm{C} 7-\mathrm{C} 8-\mathrm{C} 9-\mathrm{N} 4 \quad 53.4(2)$

$\mathrm{C} 7-\mathrm{C} 8-\mathrm{C} 9-\mathrm{C} 18 \quad-122.6(2)$

$\mathrm{C} 19-\mathrm{C} 8-\mathrm{C} 9-\mathrm{N} 4 \quad-68.7$ (2)

$\mathrm{C} 19-\mathrm{C} 8-\mathrm{C} 9-\mathrm{C} 18 \quad 115.3(2)$

$\mathrm{C} 20-\mathrm{C} 8-\mathrm{C} 9-\mathrm{N} 4 \quad 171.42(18)$

$\mathrm{C} 20-\mathrm{C} 8-\mathrm{C} 9-\mathrm{C} 18 \quad-4.6(3)$

$\mathrm{N} 4-\mathrm{C} 9-\mathrm{C} 18-\mathrm{C} 17 \quad-0.3(2)$

$\mathrm{C} 8-\mathrm{C} 9-\mathrm{C} 18-\mathrm{C} 17 \quad 176.2(2)$

$\mathrm{N} 4-\mathrm{C} 10-\mathrm{C} 11-\mathrm{C} 12 \quad 84.7$ (2)

$\mathrm{N} 4-\mathrm{C} 10-\mathrm{C} 11-\mathrm{C} 15 \quad-42.2(3)$

$\mathrm{N} 4-\mathrm{C} 10-\mathrm{C} 11-\mathrm{C} 16 \quad-159.80(18)$

$\mathrm{C} 17-\mathrm{C} 10-\mathrm{C} 11-\mathrm{C} 12 \quad-96.6(3)$

$\mathrm{C} 17-\mathrm{C} 10-\mathrm{C} 11-\mathrm{C} 15 \quad 136.6(2)$

$\mathrm{C} 17-\mathrm{C} 10-\mathrm{C} 11-\mathrm{C} 16 \quad 18.9(3)$

N4-C10- $17-\mathrm{C} 18 \quad 0.9$ (2)

$\mathrm{C} 11-\mathrm{C} 10-\mathrm{C} 17-\mathrm{C} 18 \quad-178.0$ (2) 


$\begin{array}{llll}\mathrm{C} 14-\mathrm{C} 1-\mathrm{C} 2-\mathrm{C} 3 & 46.6(2) & \mathrm{C} 10-\mathrm{C} 11-\mathrm{C} 12-\mathrm{N} 1 & -53.0(2) \\ \mathrm{C} 14-\mathrm{C} 1-\mathrm{C} 2-\mathrm{C} 27 & 172.15(17) & \mathrm{C} 10-\mathrm{C} 11-\mathrm{C} 12-\mathrm{C} 13 & -173.86(17) \\ \mathrm{C} 14-\mathrm{C} 1-\mathrm{C} 2-\mathrm{C} 28 & -71.0(2) & \mathrm{C} 15-\mathrm{C} 11-\mathrm{C} 12-\mathrm{N} 1 & 71.6(2) \\ \mathrm{N} 1-\mathrm{C} 1-\mathrm{C} 14-\mathrm{C} 13 & -18.9(2) & \mathrm{C} 15-\mathrm{C} 11-\mathrm{C} 12-\mathrm{C} 13 & -49.3(2) \\ \mathrm{C} 2-\mathrm{C} 1-\mathrm{C} 14-\mathrm{C} 13 & -148.75(17) & \mathrm{C} 16-\mathrm{C} 11-\mathrm{C} 12-\mathrm{N} 1 & -169.75(16) \\ \mathrm{C} 1-\mathrm{C} 2-\mathrm{C} 3-\mathrm{N} 2 & 108.6(2) & \mathrm{C} 16-\mathrm{C} 11-\mathrm{C} 12-\mathrm{C} 13 & 69.3(2) \\ \mathrm{C} 1-\mathrm{C} 2-\mathrm{C} 3-\mathrm{C} 26 & -73.8(3) & \mathrm{N} 1-\mathrm{C} 12-\mathrm{C} 13-\mathrm{C} 14 & -33.75(19) \\ \mathrm{C} 27-\mathrm{C} 2-\mathrm{C} 3-\mathrm{N} 2 & -17.9(3) & \mathrm{C} 11-\mathrm{C} 12-\mathrm{C} 13-\mathrm{C} 14 & 95.1(2) \\ \mathrm{C} 27-\mathrm{C} 2-\mathrm{C} 3-\mathrm{C} 26 & 159.7(2) & \mathrm{C} 12-\mathrm{C} 13-\mathrm{C} 14-\mathrm{C} 1 & 33.4(2) \\ \mathrm{C} 28-\mathrm{C} 2-\mathrm{C} 3-\mathrm{N} 2 & -134.63(19) & \mathrm{C} 10-\mathrm{C} 17-\mathrm{C} 18-\mathrm{C} 9 & -0.3(3) \\ \mathrm{C} 28-\mathrm{C} 2-\mathrm{C} 3-\mathrm{C} 26 & 43.0(3) & \mathrm{C} 7-\mathrm{C} 21-\mathrm{C} 22-\mathrm{C} 6 & -26.4(2) \\ \mathrm{N} 2-\mathrm{C} 3-\mathrm{C} 26-\mathrm{C} 25 & -1.1(2) & \mathrm{C} 4-\mathrm{C} 25-\mathrm{C} 26-\mathrm{C} 3 & 1.1(3) \\ \mathrm{C} 2-\mathrm{C} 3-\mathrm{C} 26-\mathrm{C} 25 & -179.0(2) & \mathrm{O} 1-\mathrm{C} 29-\mathrm{C} 30-\mathrm{C} 31 & 65.7(3) \\ \mathrm{N} 2-\mathrm{C} 4-\mathrm{C} 5-\mathrm{C} 6 & \mathrm{O} 1-\mathrm{C} 29-\mathrm{C} 30-\mathrm{C} 35 & -103.8(2) \\ \mathrm{N} 2-\mathrm{C} 4-\mathrm{C} 5-\mathrm{C} 23 & -51.8(3) & \mathrm{N} 1-\mathrm{C} 29-\mathrm{C} 30-\mathrm{C} 31 & -110.9(2) \\ \mathrm{N} 2-\mathrm{C} 4-\mathrm{C} 5-\mathrm{C} 24 & 69.4(2) & \mathrm{N} 1-\mathrm{C} 29-\mathrm{C} 30-\mathrm{C} 35 & 79.6(3) \\ \mathrm{C} 25-\mathrm{C} 4-\mathrm{C} 5-\mathrm{C} 6 & -172.90(18) & \mathrm{C} 29-\mathrm{C} 30-\mathrm{C} 31-\mathrm{C} 32 & -171.7(2) \\ \mathrm{C} 25-\mathrm{C} 4-\mathrm{C} 5-\mathrm{C} 23 & 143.4(2) & \mathrm{C} 35-\mathrm{C} 30-\mathrm{C} 31-\mathrm{C} 32 & -1.6(3) \\ \mathrm{C} 25-\mathrm{C} 4-\mathrm{C} 5-\mathrm{C} 24 & -95.5(3) & \mathrm{C} 29-\mathrm{C} 30-\mathrm{C} 35-\mathrm{C} 34 & 169.84(19) \\ \mathrm{N} 2-\mathrm{C} 4-\mathrm{C} 25-\mathrm{C} 26 & 22.3(3) & \mathrm{C} 31-\mathrm{C} 30-\mathrm{C} 35-\mathrm{C} 34 & 0.4(3) \\ \mathrm{C} 5-\mathrm{C} 4-\mathrm{C} 25-\mathrm{C} 26 & -0.5(2) & \mathrm{C} 30-\mathrm{C} 31-\mathrm{C} 32-\mathrm{C} 33 & 1.6(3) \\ \mathrm{C} 4-\mathrm{C} 5-\mathrm{C} 6-\mathrm{N} 3 & 166.1(2) & \mathrm{C} 31-\mathrm{C} 32-\mathrm{C} 33-\mathrm{C} 11 & 177.89(18) \\ \mathrm{C} 4-\mathrm{C} 5-\mathrm{C} 6-\mathrm{C} 22 & 48.0(2) & \mathrm{C} 31-\mathrm{C} 32-\mathrm{C} 33-\mathrm{C} 34 & -0.4(3) \\ \mathrm{C} 23-\mathrm{C} 5-\mathrm{C} 6-\mathrm{N} 3 & -71.3(2) & \mathrm{C} 11-\mathrm{C} 33-\mathrm{C} 34-\mathrm{C} 35 & -179.02(17) \\ \mathrm{C} 23-\mathrm{C} 5-\mathrm{C} 6-\mathrm{C} 22 & -72.3(2) & \mathrm{C} 32-\mathrm{C} 33-\mathrm{C} 34-\mathrm{C} 35 & -0.8(3) \\ \mathrm{C} 24-\mathrm{C} 5-\mathrm{C} 6-\mathrm{N} 3 & 168.44(17) & \mathrm{C} 33-\mathrm{C} 34-\mathrm{C} 35-\mathrm{C} 30 & 0.7(3) \\ & 170.03(16) & & \end{array}$

Hydrogen-bond geometry $\left(A,{ }^{\circ}\right)$

$\mathrm{Cg} 1$ is the centroid of pyrrole ring N2/C3/C4/C25/C26; $\mathrm{Cg} 2$ is the centroid of the benzene ring $\mathrm{C} 30-\mathrm{C} 35$.

\begin{tabular}{lllll}
\hline$D-\mathrm{H} \cdots A$ & $D-\mathrm{H}$ & $\mathrm{H} \cdots A$ & $D \cdots A$ & $D-\mathrm{H} \cdots A$ \\
\hline $\mathrm{N} 2-\mathrm{H} 2 \cdots \mathrm{N} 3$ & 0.88 & 2.31 & $2.865(2)$ & 121 \\
$\mathrm{~N} 4-\mathrm{H} 4 \cdots \mathrm{N} 3$ & 0.88 & 2.55 & $3.051(2)$ & 117 \\
$\mathrm{C} 15-\mathrm{H} 15 A \cdots \mathrm{N} 2$ & 0.98 & 2.52 & $3.488(3)$ & 171 \\
$\mathrm{C} 15-\mathrm{H} 15 A \cdots C g 1$ & 0.98 & 2.40 & $3.301(2)$ & 152 \\
$\mathrm{~N} 3-\mathrm{H} 3 N \cdots \mathrm{O} 1^{\mathrm{i}}$ & $0.882(18)$ & $2.257(18)$ & $3.105(2)$ & $161.1(18)$ \\
$\mathrm{C} 23-\mathrm{H} 23 B \cdots \mathrm{O} 1^{\mathrm{i}}$ & 0.98 & 2.53 & $3.495(3)$ & 168 \\
$\mathrm{C} 27-\mathrm{H} 27 C \cdots C g 2^{\mathrm{i}}$ & 0.98 & 2.82 & $3.702(2)$ & 150 \\
\hline
\end{tabular}

Symmetry code: (i) $-x+3 / 2, y-1 / 2,-z+1 / 2$. 